\title{
Réformes hospitalières et crise pandémique de la Covid-19 : depuis 2008 les inégalités spatiales d'équipement en lits de réanimation ont-elles augmenté ?
}

Reforms and sanitary covid crisis: have spatial inequalities in resuscitation beds increased since 2008?

Benoit Conti, Sophie Baudet-Michel et Charlène Le Neindre

\section{(2) OpenEdition}

Journals

Édition électronique

URL : http://journals.openedition.org/rfst/909

DOI : $10.4000 /$ rfst.909

ISSN : 2492-3672

Éditeur

Espaces et SOciétés (UMR 6590)

Référence électronique

Benoit Conti, Sophie Baudet-Michel et Charlène Le Neindre, « Réformes hospitalières et crise pandémique de la Covid-19: depuis 2008 les inégalités spatiales d'équipement en lits de réanimation ont-elles augmenté ?», Revue francophone sur la santé et les territoires [En ligne], Pandémie, crises et perspectives : lectures territoriales de la Covid-19, mis en ligne le 12 mars 2021, consulté le 06 avril 2021. URL : http://journals.openedition.org/rfst/909 ; DOI : https://doi.org/10.4000/rfst.909

Ce document a été généré automatiquement le 6 avril 2021.

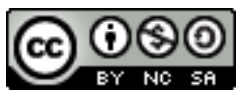

La Revue francophone sur la santé et les territoires est mise à disposition selon les termes de la Licence Creative Commons Attribution - Pas d'Utilisation Commerciale - Partage dans les Mêmes Conditions 4.0 International. 


\section{Réformes hospitalières et crise pandémique de la Covid-19: depuis 2008 les inégalités spatiales d'équipement en lits de réanimation ont-elles augmenté ?}

Reforms and sanitary covid crisis: have spatial inequalities in resuscitation beds increased since 2008?

Benoit Conti, Sophie Baudet-Michel et Charlène Le Neindre

Nous remercions les deux relecteurs pour leurs remarques et suggestions : elles nous ont aidé à préciser et améliorer le contenu de notre article.

\section{Introduction}

1 L'épidémie de Covid-19 a débuté en France en février 2020. Depuis le début de celle-ci et jusqu'à ce jour (8 février 2021), 3,3 millions de cas confirmés ont été enregistrés et plus de 790000 décès sont survenus ${ }^{1}$. Alors que l'épidémie est repartie à la hausse depuis le début de l'automne 2020, la deuxième vague épidémique est en cours de stagnation depuis le début du mois de décembre avec plus de 28000 malades hospitalisés dont 3 300 en réanimation ${ }^{2}$ (8 février). L'évolution du nombre de personnes en réanimation a fluctué au moment du premier pic épidémique en avril 2020, pour atteindre 7000 personnes ; elle a atteint plus de 4900 personnes lors du second à la mi-novembre 2020.

Dans la nuit du 14 au 15 mars 2020, la pandémie est passée du stade 2 (freiner la propagation du virus sur le territoire) au stade 3 (atténuer les effets de la vague épidémique). Un plan de confinement général de l'ensemble de la population résidant sur le territoire français a été mis en place (à partir du mardi 17 mars 2020). Un des objectifs du confinement était de freiner la propagation de l'épidémie pour limiter la 
pression sur les services hospitaliers et les unités de réanimation. Ces dernières prennent en charge les patients critiques jusqu'à ce que leurs fonctions vitales ne soient plus menacées (Andréo, 2004). Dans la situation de crise qu'a déclenchée l'apparition de la pandémie, différents pays ont transformé leurs lits de soins intensifs ${ }^{3}$ en lits de réanimation afin d'absorber le flux rapide de malades. Au début de l'épidémie, la France disposait de 5000 lits de réanimation, soit une densité de 7,5 lits pour 100000 habitants. 3000 lits de plus ont été obtenus par la transformation des lits de soins intensifs et de surveillance continue ${ }^{4}$ (Gandré \& Or, 2020). À titre de comparaison, l'Allemagne comptait en 2017, 28031 lits d'hospitalisation de soins intensifs (dont 25 000 avec des capacités de ventilation), soit 33,7 lits pour 100000 habitants, cinq fois plus qu'en France. La capacité limitée à créer des lits de réanimation temporaires en France a conduit certaines Agences Régionales de Santé à pratiquer des transferts de patients vers des pays ou des régions moins affectées (644 patients ont été concernés du 18 mars au 9 avril). Dans la seule région Grand-Est-Bourgogne-Franche-Comté, 317 patients ont été concernés entre le 15 mars 2020 et le 10 avril 2020 (Tritsch et al., 2020).

Depuis désormais plusieurs décennies, et particulièrement depuis 20 ans, le système hospitalier enregistre des réformes qui visent à améliorer la "productivité" hospitalière et qui ont surtout abouti à la réduction des capacités d'accueil hospitalières tant en termes d'infrastructure que de personnel soignant, d'autant plus lorsqu'on les envisage au regard d'une augmentation de la population, et particulièrement du nombre de personnes âgées. Cette situation de réforme et de réduction des moyens est devenue la norme. Elle perdure depuis 20 ans en dépit des protestations des médecins et personnels hospitaliers. La France n'est pas la seule à enregistrer ces réformes, et les systèmes de soins de la plupart des pays de l'OCDE $^{5}$ ont subi des transformations similaires (Vigneron \& Haas, 2009). Parmi ces changements, de nombreuses fermetures de lits, de services ou même d'hôpitaux, orchestrées au niveau régional par les Agences Régionale de Santé, ont inégalement affecté les villes et régions françaises (Baillot \& Evain, 2013 ; Vigneron, 2017). Lucas-Gabrielli et Tonnelier (2000) ont montré que les fermetures de lits ont commencé dès les années 1990. Depuis 2000, ces fermetures se sont poursuivies dans la quasi-totalité des villes (Conti et al., 2020), allant jusqu'à la disparition totale pour une soixantaine d'entre elles. La diminution des lits de courts séjours est bien connue (Lucas-Gabrielli \& Tonnelier, 2000 ; Conti et al., 2020), la façon dont les réformes hospitalières ont modifié les capacités hospitalières en réanimation l'est moins. Or cet équipement a été particulièrement essentiel pour répondre aux besoins de soins pendant la crise sanitaire.

4 L'objectif de cet article est de proposer une analyse de l'évolution de la géographie de l'équipement en lits de réanimation à deux niveaux : celui des régions d'une part et celui du système urbain d'autre part; et dans deux situations : au moment de la crise épidémique et dans le contexte de la dernière décennie de réforme hospitalière. À partir de l'exploitation de la Statistique Annuelle des Établissements de santé (SAE), qui renseigne sur le nombre de lits de réanimation, de soins intensifs et de surveillance continue sur la période 2008-2018, nous analyserons l'évolution de l'organisation spatiale de l'équipement de réanimation sur le territoire de la France métropolitaine en croisant échelles d'espace et situations de crise ou de réforme. Avant de présenter les résultats, une mise en perspective de l'évolution de l'équipement hospitalier en lien avec les réformes législatives sera proposée. Celle-ci fournira un cadre d'analyse plus 
large dans lequel s'insère la problématique d'évolution de la distribution spatiale des lits de réanimations en France.

\section{Conséquences territoriales de la baisse de lits de court séjour}

\section{La diminution du nombre de lits : une situation de réforme, en cours depuis une quarantaine d'années}

5 Au cours des 40 dernières années, le nombre de lits d'hospitalisation de courte durée a diminué, et ce malgré un contexte de croissance démographique. Cette évolution s'explique à la fois par le passage à des formes d'hospitalisation ambulatoire de moins de $24 \mathrm{~h}$ (Granger \& Pierru, 2012), mais aussi par la mise en place de réformes hospitalières d'inspiration managériale (Belorgey, 2010; Domin, 2015; Belorgey \& Pierru, 2017). En France dans les années 1980, alors que la part de la dépense hospitalière est à son point le plus élevé ( $52 \%$ des dépenses de soins et biens médicaux en 1982), une conception entrepreneuriale de l'hôpital va influencer les politiques hospitalières et déclencher une réduction progressive du nombre de lits d'hospitalisation. Sous l'égide des Agences Régionales d'Hospitalisation (ARH) puis des Agences Régionales de Santé (ARS) ${ }^{6}$, les Schémas Régionaux d'Organisation Sanitaire (SROS) vont fixer tous les 5 ans le nombre d'installations et d'activités sanitaires ${ }^{7}$ privées et publiques selon des découpages de l'espace en secteurs sanitaires ${ }^{8}$, puis en territoires de santé (Delas, 2011). Si les premiers SROS (1991) avaient pour objectif de moderniser l'offre de soins et de l'adapter aux besoins de la population au niveau des régions, à partir des années 2000 , les objectifs de réduction des dépenses de santé deviennent une priorité (Lerouvillois \& Vinclet, 2002). Les établissements hospitaliers doivent désormais répondre à des objectifs de maîtrise des dépenses ${ }^{9}$. Les ARS qui organisent l'offre hospitalière au niveau régional ont tendance à concentrer les activités dans un nombre restreint de grands établissements et à spécialiser les établissements, afin notamment de réduire les coûts de «production » des soins, mais aussi pour assurer un certain niveau de qualité et de sécurité de certains soins. Ainsi, certaines fermetures d'établissements ou de services peuvent être déclenchées par une faible activité de soins, elle-même liée au déclin démographique du territoire environnant, à des difficultés à recruter du personnel soignant, au choix des patients pour d'autres structures de soins. Ces transformations managériales accompagnent une transformation technique, le déploiement important d'une offre d'hospitalisation de très courte durée (moins de $24 \mathrm{~h}$ ) à travers la création de places d'hospitalisations. Ces transformations ne sont pas propres à la France, et s'inscrivent à la fois dans une transformation technologique et dans une nouvelle manière de penser l'action publique, le New Public Management (NPM) ${ }^{10}$.

\section{Le passage à l'ambulatoire : un phénomène répandu dans les pays de l'OCDE}

6 La plupart des États de l'OCDE se sont inspirés des principes du NPM pour penser de nouvelles politiques de santé et mettre en place des réformes des systèmes hospitaliers. Selon l'OCDE ${ }^{11}$, la crise économique de 2008 a accentué la volonté de réduire les 
dépenses publiques de soins. Entre 1980 et 2015, le nombre total de lits d'hôpitaux a baissé de $48 \%$ dans les pays de l'OCDE. Le nombre de lits par habitant a diminué dans l'espace européen depuis les années 1980, passant de 900 lits pour 100000 habitants dans les États d'Europe de l'Ouest à 564 pour 100000 habitants en 2005 (Vas et al., 2008). Entre 2000 et 2017, la baisse a été moins forte en Allemagne (de 919 à 800 lits pour 100000 habitants [-13\%]) et plus importante en France (de 797 à 591 [-26\%]), en Italie (de 471 à 314 [-33\%]), au Royaume-Uni (de 408 à 254 [-38 \%]) et en Suède (de 358 à 214 [-40\%]). En France métropolitaine, le nombre de lits de court séjour ${ }^{12}$ a diminué de $15 \%$, passant de 241275 à 204520 entre 2000 et 2016, tandis que le nombre d'habitants augmentait de $10 \%$ de 58,9 à 64,5 millions (Conti et al., 2020). La densité de lits de court séjour par habitant a ainsi diminué de $23 \%$, passant de 409 à 317 lits pour 100000 habitants.

7 Dans le même temps, dans tous les pays de l'OCDE, une transformation radicale s'est opérée dans les modes d'hospitalisation. Connue sous le nom de passage à l'ambulatoire, cette transformation a permis à des patients, notamment en chirurgie, de rentrer et sortir de l'hôpital dans la journée, répondant ainsi à leurs souhaits de réduire leur temps d'hospitalisation. La transformation ambulatoire a indéniablement permis d'améliorer la qualité de certains soins (par exemple les opérations de la cataracte) et le retour rapide à domicile des patients pour la convalescence. Elle a été possible en raison des progrès techniques accomplis dans les domaines de l'anesthésie et de la chirurgie, permettant de pratiquer des soins moins invasifs. Cette transformation technologique a ainsi participé à l'augmentation de la "productivité" hospitalière, permettant de conduire davantage d'opérations, surtout de chirurgie, dans un temps plus réduit. En termes d'équipement, elle s'est traduite par une augmentation des places d'accueil pour des durées de moins de $24 \mathrm{~h}$. En France par exemple, le nombre de places a augmenté de 15509 en 2000 à 31645 en 2016, multipliant par deux la densité de places de 26,3 à 49 pour 100000 habitants (BaudetMichel et al., 2019). Mais tandis que le nombre de places augmentait, le nombre de lits, permettant des hospitalisations pour des durées supérieures à plus de $24 \mathrm{~h}$ diminuaient considérablement. Cette diminution, en situation de crise sanitaire, a considérablement handicapé la capacité d'accueil pour des durées supérieures à quelques jours. Or, pendant la crise sanitaire, les temps d'hospitalisation des patients en réanimation ont souvent été d'au moins 14 jours (Eichwald \& Moreau, 2020).

\section{La diminution du nombre de lits : porteuse d'inégalités territoriales?}

En Nouvelle-Zélande, Barnett (1999) a étudié la restructuration du secteur des soins dans les années 1990. Il a montré que la stratégie de réduction des coûts a eu pour conséquence la fermeture ou la diminution des capacités des services hospitaliers, particulièrement dans les périphéries rurales et les régions du Sud. En Italie, les réformes hospitalières ont davantage ciblé les régions où les déficits financiers en santé étaient importants, amenant des fermetures d'hôpitaux et de services (Perucca et al., 2019). Ces restructurations ciblées ont renforcé les inégalités: De Belvis et al. (2012) montrent que la région italienne de Campanie a été particulièrement ciblée par les réformes alors que l'état de santé de la population est un des moins bons du pays. Franzini et Giannoni (2010) font le même constat pour la Sicile. Dans les Alpes italiennes, de nombreux hôpitaux ont été fermés et la fréquentation des hôpitaux restants a diminué (De Belvis et al., 2012 ; Perucca et al., 2019). Carrière et al. (2020) et 
Liu et al. (2001) ont montré les mêmes effets de diminution de la fréquentation hospitalière suite à des fermetures de lits dans les provinces peu denses du Manitoba et du Saskatchewan (Canada).

En France, plusieurs études quantitatives montrent que les petites villes ont été particulièrement impactées par les fermetures de services ou établissements (Jousseaume, 2002 ; Baillot \& Evain, 2013; Barczak \& Hilal, 2017). Conti et al. (2020) montrent que 36000 lits de court séjour ont été fermés entre 2000 et 2016, affectant $75 \%$ des villes équipées (sur un échantillon de 539 villes équipées au moins une fois sur la période). La densité médiane de lits de court séjour est passée de 540 à 440 lits pour 100000 habitant. Les grandes villes les plus équipées - en termes de volume - ont perdu les plus grandes quantités de lits (fermeture de 11000 lits à Paris, soit $1 / 3$ des fermetures, pour un niveau d'équipement de 46623 lits en 2000), et une soixantaine de villes (par exemple La Flèche, Cadenet, Lectour, Saint-Mihiel) a totalement perdu cet équipement.

Au niveau régional, la diminution du nombre de lits a pu s'accompagner d'un phénomène de convergence : Lucas-Gabrielli et Tonnellier (2000) ont montré pour la période 1987-1997 que la réduction du nombre de lits avait entraîné une réduction des écarts initiaux et une convergence inter-régionale en termes de densité de lits: les diminutions ont été les plus fortes dans les régions les plus équipées en 1987 et plus faibles dans les moins équipées. De même, les fermetures de maternités et de lits d'obstétrique et leur redistribution se sont accompagnées d'une réduction des disparités inter-régionales de lits par accouchement (nombre de lits rapporté au nombre d'accouchements) (Baillot \& Evain, 2013). En Europe centrale et orientale, les pays où l'offre de départ était plus forte ont enregistré des rythmes de fermetures plus élevés entre 2007 et 2017 : la Hongrie et la République Tchèque par exemple (DubasJakóbczyk et al., 2020).

\section{Qu'en est-il pour les lits de réanimation? Problématique et hypothèses}

11 Si les conséquences territoriales des réformes hospitalières sur la réorganisation territoriale des lits de court séjour sont connues, le cas des lits de réanimation l'est moins. Très mobilisées durant les pics épidémiques de la Covid-19 (mois de mars à mai 2020, et depuis le mois d'octobre), les capacités d'accueil des services de réanimation constituent un enjeu majeur pour les régions touchées par la pandémie. Ces capacités ont été conditionnées par la présence des soignants, mais aussi par le nombre de lits de réanimation, dans un contexte où les patients gravement atteints par la maladie restaient hospitalisés en réanimation pour des durées supérieures (en moyenne 14 jours ${ }^{13}$ ) à la durée moyenne de séjour de 7,2 jours (Carpentier et al., 2015). Si l'État et certains journaux nationaux en ligne proposent des analyses spatialisées de l'offre de soins de réanimation ${ }^{14}$, leurs évolutions sont peu connues. Comment la répartition de l'équipement de réanimation a-t-elle évolué dans le contexte de réforme de la dernière décennie ? Des lits de réanimation ont-ils été fermés et si oui où ?

12 Cet article expose la manière dont s'est réorganisé spatialement l'équipement en lits de réanimation sur le territoire de la France métropolitaine. Nous proposons ici une lecture à deux échelles (régions et système urbain) et pour deux types de situations : 
une situation décennale de réformes hospitalières, une situation ponctuelle de crise sanitaire. Dans la dernière décennie de poursuite des réformes hospitalières, comment a évolué la répartition de l'équipement en lits de réanimation dans l'espace des régions et des villes ? Y a-t-il eu convergence de l'équipement en lits de réanimation, et, si oui, à quelle échelle? Dans la période de crise de la pandémie survenue en 2020, les médecins hospitaliers et particulièrement les services de réanimation ont fait la preuve de leurs capacités à adapter les équipements dont ils disposaient ${ }^{15}:$ des lits de soins intensifs et de surveillance continue ont été transformés en lits de réanimation. Quel était le niveau d'équipement en soins intensifs et de surveillance continue au début de la pandémie, et comment cet équipement pouvait-il augmenter les capacités d'accueil localement? Trois hypothèses guident notre démonstration :

1. Pour ce qui concerne la situation de réforme des 10 dernières années, nous faisons l'hypothèse que l'évolution de l'équipement en lits de réanimation va dans le sens d'une diminution généralisée en termes de densités, tant au niveau des régions que des villes.

2. À ces deux échelles et pour ce qui concerne la situation de réforme des dernières années, nous faisons l'hypothèse d'une convergence inter-régionale et inter-urbaine des densités.

3. Dans la situation de crise de la pandémie, nous faisons l'hypothèse que le potentiel de transformation des lits de soins intensifs et de surveillance continue en lits de réanimation diffère fortement entre les régions et les villes.

\section{Sources et méthodes}

\section{Sources : la statistique annuelle des établissements (SAE) et le recensement de la population}

Deux échelles d'observation et d'analyse sont mobilisées : celle des régions (ancien découpage) et des aires urbaines ${ }^{16}$ (zonage 2010). Nous retenons l'ancien découpage régional, qui a continué d'être opératoire entre 2009 et 2015, avant la réforme territoriale qui a réduit le nombre de régions métropolitaines de 22 à 13 . C'est à l'échelle des régions que les ARS organisent, depuis la loi Hôpital Patient Santé Territoires (2009), la répartition des équipements hospitaliers à travers le système des accréditations et autorisations d'exercices. L'échelle des aires urbaines permet de mettre en lumière l'évolution plus fine de la localisation d'un équipement rare et de haut niveau (les lits de réanimation) même si le périmètre de desserte de celui-ci dépasse les limites fonctionnelles des villes. Les données mobilisées, concernant l'équipement de lits de réanimation, sont issues d'un traitement ad hoc de la base statistique de la $\mathrm{SAE}^{17}$ (Statistique Annuelle des Établissements), fournie par le Ministère de la Santé et notamment par la DREES (Direction de la recherche, des études, de l'évaluation et des statistiques).

14 La situation de réforme est analysée sur la période 2008-2018. En effet, 2008 est l'année la plus ancienne pour laquelle les données de nombre de lits de réanimation sont disponibles ${ }^{18} ; 2018$, car il s'agit de l'année la plus récente au moment de la rédaction de cet article. La construction d'une base de données longitudinale a été nécessaire afin de pallier certaines transformations de la SAE (voir Annexe). D'autres données, fournies par l'Insee (Institut national de la statistique et des études économiques), issues du recensement de la population ${ }^{19}$, sont mobilisées afin de mettre en regard le nombre d'habitants (en 2008 et 2016) et le niveau d'équipement de lits de réanimation. Pour la 
période de crise sanitaire, les données de la SAE de l'année 2018 sont mobilisées. Le fichier contenant les informations sur les lits adultes de réanimation, de soins intensifs et de surveillance continue est disponible au niveau de l'ensemble des structures de soins; il permet de connaitre, pour chaque structure de soin (établissement géographique) l'équipement existant.

\section{Les indicateurs et les méthodes quantitatives mobilisés}

À partir d'un travail d'agrégation des informations disponibles au niveau des régions et des aires urbaines, plusieurs indicateurs sont calculés pour l'analyse de la situation de réforme :

- Le volume de lits de réanimation dans les régions et les aires urbaines en 2008 et 2018

- La densité de lits de réanimation dans les régions et les aires urbaines en 2008 (population de 2008) et en 2018 (population de 2016)

- Les taux de variations du nombre de lits de réanimation et de la densité de lits de réanimation dans les régions et les aires urbaines pour la période 2008-2018.

Pour l'analyse de la situation de crise de 2020, les volumes de lits de soins intensifs et de surveillance continue en 2018 sont mobilisés. Pour les régions, l'ensemble des lits de surveillance et de soins intensifs disponibles dans la région est considéré et mis au regard de l'ensemble des lits de réanimation. Au niveau des aires urbaines, nous avons pris en compte l'ensemble des lits de surveillance et de soins intensifs disponibles dans les aires urbaines ayant au moins un lit de réanimation en $2018^{20}$. La diversité des actions de transformation des lits de surveillance ou de soins intensifs engagées par les équipes soignantes nous a conduits à élaborer un potentiel théorique de transformations (nommé Pt); il rapporte la somme des lits de soins intensifs et de surveillance continue au nombre de lits de réanimation pour chaque région et chaque aire urbaine.

\section{$P t=\frac{\text { Nombre de lits de soilnsintensifs }+ \text { Nombre de lits de surveillance continue }}{\text { Nombre de lits de réanimation }} \times 100$}

Ce potentiel est exprimé en pourcentage : plus le pourcentage est fort, plus le potentiel de transformation est important, plus les ressources en lits transformables (de soins intensifs et de surveillance continue) sont élevées au regard des ressources en lits de réanimation. Par exemple un potentiel de transformation de $100 \%$ signifie que le nombre de lits de réanimation de l'aire urbaine peut être doublé par transformation des lits de soins intensifs et de surveillance continue. Cet indicateur est purement théorique et ne s'appuie pas sur la réalité de la transformation opérée au printemps 2020.

18 Afin de tester les hypothèses, plusieurs méthodes sont mobilisées. Pour répondre à l'hypothèse 1 (diminution généralisée), une analyse de l'évolution des paramètres de tendance centrale (minimum, maximum, moyenne, médiane) des indicateurs de volume, de densité et de taux d'évolution des lits de réanimation est réalisée. Pour l'hypothèse 2 (convergence), deux méthodes sont produites : (i) analyse de l'évolution du coefficient de variation, des indicateurs de volume, de densité et de taux d'évolution des lits de réanimation ; (ii) régression linéaire entre la densité initiale de lits et le taux de variation de l'équipement sur la période. Enfin, pour l'hypothèse 3 (potentiel différencié), deux méthodes sont aussi mobilisées: (i) analyse de l'évolution des paramètres de tendance centrale et de dispersion de l'indicateur de volume des lits de 
surveillance continue et de soins intensifs ; (ii) régression linéaire entre la densité de lits de réanimation en 2018 et le potentiel de transformation.

\section{Hypothèse 1. 2008_2018 : en situation de réforme, diminution de l'équipement : baisse au niveau régional et perte d'équipement pour une vingtaine de villes}

Depuis 2008, en France métropolitaine, le nombre de lits de réanimation est resté quasi stable passant de 4983 à 4948 lits $(-0,7 \%)$ (a). En moyenne, les régions ont gardé un niveau d'équipement assez stable, de 227 à 225 lits, et la Corse, région la moins équipée, a perdu 2 lits, tandis que l'Île-de-France, région la plus équipée, en a perdu 28. Les disparités inter-régionales se sont conservées avec un coefficient de variation stable à $103 \%$.

Tableau $1 \mathrm{a}$ - Paramètres de tendance centrale et de dispersion du nombre de lits de réanimation dans les régions en 2008 et en 2018 et leurs évolutions

\begin{tabular}{|l|c|c|c|}
\hline & 2008 & 2018 & Solde \\
\hline Nombre de régions équipées & 22 & 22 & 0 \\
\hline Somme de lits & 4983 & 4948 & -35 \\
\hline Minimum (Corse) & 22 & 18 & -4 \\
\hline Maximum (Ile-de-France) & 1181 & 1153 & -28 \\
\hline Moyenne & 227 & 225 & -2 \\
\hline Médiane & 173 & 171 & -2 \\
\hline Écart-type & 234 & 231 & -3 \\
\hline Coefficient de variation & $103 \%$ & $103 \%$ & 0 \\
\hline $\begin{array}{l}\text { N. B. Les valeurs calculées pour les deux années sont produites à partir des régions équipées (au } \\
\text { moins un lit) }\end{array}$ & & \\
\hline
\end{tabular}

\section{Sources : DREES, Statistique annuelle des établissements, exploitation des auteurs}

Cependant des dynamiques régionales distinctes apparaissent: douze régions ont supprimé un total de 196 lits alors que les dix autres ont ouvert 161 lits de réanimation supplémentaires ${ }^{21}$. Les régions les plus concernées par les baisses sont la Lorraine (- 44 lits), l'Alsace (-43 lits) et l'île-de-France (-28 lits). Ces régions ont été particulièrement touchées par la Covid-19 au printemps 2020. Le Languedoc-Roussillon, moins touché, appartient aussi à ce groupe (-27 lits). D'autres régions, au contraire, ont connu une augmentation: Midi-Pyrénées (+42 lits), Aquitaine (+34 lits), Nord-Pas-de-Calais (+28 lits) et Rhône-Alpes (+21 lits). Ces évolutions brutes sont à mettre en regard d'un stock initial très variable d'une région à l'autre, fonction de la population régionale : avec 1153 lits de réanimation en 2018, l'île-de-France reste la plus équipée ${ }^{22}$ tandis que la Corse (18 lits) et le Limousin (47 lits) offrent peu de lits ${ }^{23}$.

21 Pour l'ensemble du système urbain, au cours de la dernière décennie, les niveaux moyens (de 29 à 33 lits) et médians d'équipement (de 10 à 12 lits) augmentent (tableau 
1b). La baisse du coefficient de variation signale en outre une diminution des disparités inter-urbaines pour l'échantillon des villes équipées. L'équipement de réanimation est rare puisque $40 \%$ seulement des villes équipées en lits d'hospitalisation de courte durée (400 aires en 2018 [Conti et al., 2020]) offrent des lits de réanimation. Toutefois, en dépit de la diminution des inégalités observées, la concentration de l'équipement de réanimation a augmenté puisque le nombre d'aires équipées a diminué de 169 à 150 . Cet équipement, déjà rare en 2008, sélectionne encore plus fortement les villes en 2018: 21 aires urbaines $(12 \%)$ ont perdu la totalité de leur l'équipement initial, tandis que 2 villes $^{24}$ seulement ont gagné l'équipement. Les aires urbaines concernées par les fermetures ${ }^{25}$ sont généralement petites, proches d'aires urbaines équipées et très présentes dans l'est de la France.

Tableau $1 \mathrm{~b}$ - Paramètres de tendance centrale et de dispersion du nombre de lits de réanimation dans les aires urbaines équipées en 2008 et en 2018 et leurs évolutions

\begin{tabular}{|l|c|c|c|}
\hline & 2008 & 2018 & Solde \\
\hline Nombre d'AU équipées (avec au moins un lit) & 169 & 150 & -19 \\
\hline Somme de lits dans les AU équipées & 4983 & 4923 & -60 \\
\hline Minimum (AU de Mende [2018]) & 4 & 3 & -1 \\
\hline Maximum (AU de Paris) & 1175 & 1145 & -30 \\
\hline Moyenne & 29 & 33 & +4 \\
\hline Médiane & 10 & 12 & +2 \\
\hline $1^{\text {er décile }}$ & 6 & 8 & +2 \\
\hline $9^{\text {e décile }}$ & 58 & 64 & +6 \\
\hline Écart-type & 96 & 99 & +3 \\
\hline Coefficient de variation & $324 \%$ & $302 \%$ & -22 \\
\hline $\begin{array}{l}\text { N. B. Les valeurs calculées pour les deux années sont produites à partir des aires équipées (au } \\
\text { moins un lit) }\end{array}$ \\
\hline
\end{tabular}

Sources : DREES, Statistique annuelle des établissements, exploitation des auteurs

Quelques grandes aires urbaines ont aussi enregistré au cours des 12 dernières années une baisse d'une trentaine de lits ${ }^{26}$ tandis que d'autres ont bénéficié d'une augmentation d'une quinzaine à une cinquantaine de lits ${ }^{27}$. Parmi celles qui ont perdu, notamment Paris, Marseille et Metz ont été sous tension au moment du premier pic épidémique si bien que certains patients ont dû être évacués vers d'autres hôpitaux: ainsi en région Grand Est en mars 2020, 320 patients ont été évacués vers d'autres régions par manque de lits et de personnels de réanimation (Eichwald \& Moreau, 2020). 


\section{Hypothèse 2. En situation de réforme : une convergence par le bas des densités de lits au niveau des régions et des aires urbaines}

L'examen des densités de lits de réanimation par habitant atteste de diminutions. En effet, si le nombre de lits de réanimation est resté stable au niveau national depuis 2008 , la population a augmenté de 62,1 millions en 2008 à 64,5 millions au niveau des régions et de 45,5 millions à 46,7 millions pour les aires équipées entre 2008 et 2016.

À l'échelle des régions, échelle de planification des équipements de soins, la densité moyenne de lits de réanimation a baissé de 7,7 à 7,3 lits pour 100000 habitants sur la période (tableau 2a). Entre 2008 et 2018, les réductions de l'écart-type, du coefficient de variation, de l'écart interdécile attestent d'une forme de convergence inter-régionale. La convergence opère à la baisse puisque les densités médianes et moyennes de lits pour 100000 habitants baissent. 15 régions sur 22 enregistrent une évolution négative de leur densité de lits allant jusqu'à $-25 \%$ en Corse, $-20 \%$ en Alsace, $-19 \%$ en Lorraine ${ }^{28}$. Seules huit régions enregistrent des hausses de densités (Haute-Normandie, Aquitaine, Nord Pas-de-Calais, Haute et Basse Normandie, Limousin, Midi-Pyrénées, FrancheComté).

Tableau 2a - Paramètres de tendance centrale et de dispersion de la densité de lits de réanimation (nombre de lits pour 100000 habitants) dans les régions en 2008 et en 2018 et leurs évolutions

\begin{tabular}{|l|c|c|c|}
\hline & 2008 & 2018 & Solde \\
\hline Nombre de régions équipées & 22 & 22 & 0 \\
\hline Minimum (Pays de la Loire [2018]) & 5,1 & 4,8 & $-0,3$ \\
\hline Maximum (Alsace) & 12,8 & 10,2 & $-2,6$ \\
\hline Moyenne & 7,7 & 7,3 & $-0,4$ \\
\hline Médiane & 7,2 & 7,1 & $-0,1$ \\
\hline $1^{\text {er décile }}$ & 5,3 & 5,5 & $+0,2$ \\
\hline $9^{\text {e décile }}$ & 9,8 & 9,2 & $-0,6$ \\
\hline Écart-type & 1,9 & 1,5 & $-0,4$ \\
\hline Coefficient de variation & $24 \%$ & $21 \%$ & -3 \\
\hline
\end{tabular}

Sources : DREES, Statistique annuelle des établissements \& INSEE, recensement, exploitation des auteurs 
Carte 1a - Taux d'évolution de la densité de lits de réanimation dans les régions entre 2008 et 2018

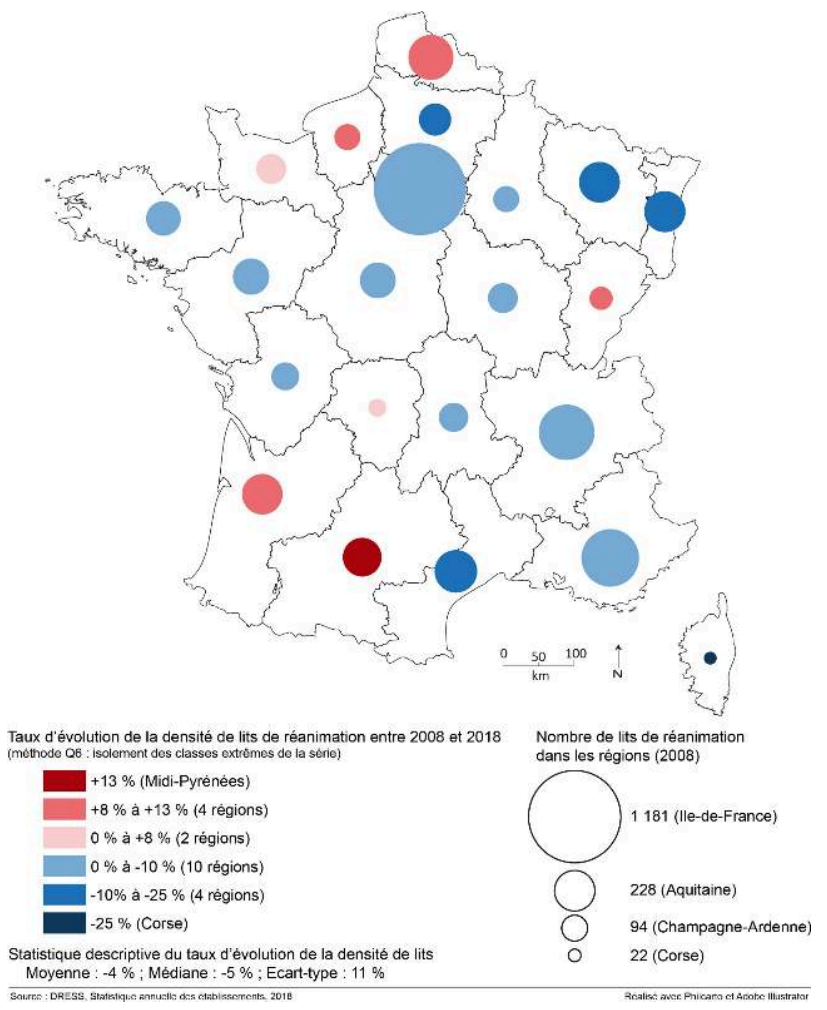

Au niveau des aires urbaines aussi, la densité de lits de réanimation a diminué passant de 14,5 à 13,1 lits en moyenne pour 100000 habitants (Tableau 3b). Le niveau médian d'équipement a baissé de 12,2 à 11,7 lits pour 100000 habitants. 102 des 169 aires urbaines équipées en 2008 ont vu leur densité de lits chuter (Carte 1b) et la carte illustre l'ubiquité du phénomène. Dans de nombreuses régions, les plus grandes villes ont enregistré une diminution des densités de lits de réanimation (Lorraine, Alsace, Languedoc-Roussillon, Rhône-Alpes, Bretagne, PACA) et dans la plupart des régions, plus de $70 \%$ des villes ont enregistré des baisses. La baisse moyenne a été de - $12 \%$ par ville et la moitié des villes ont enregistré une baisse d'au moins - $3 \%$. Pour certaines villes, la baisse a été très prononcée : Montpellier $(-26 \%)$, Nancy $(-27 \%)$, Thionville ($35 \%)$, Aurillac $(-47 \%)$ ou Montluçon $(-48 \%)$. Dans un contexte de très légère diminution du nombre de lits (Tableau 1b), c'est d'abord l'augmentation de la population des aires urbaines qui explique mécaniquement les situations de baisse de densité de lits de réanimation.

Tableau 3b - Paramètres de tendance centrale et de dispersion des densités de lits de réanimation (lits/100 000 habitants) dans les aires urbaines en 2000 et 2018 et du taux d'évolution de cette densité de lits.

\begin{tabular}{|l|c|c|c|}
\hline & 2008 & 2018 & Taux d'évolution \\
\hline Nombre d'AU équipées (avec au moins un lit) & 169 & 150 & $-11 \%$ \\
\hline Minimum (AU Béthune) & 2,5 & 3,0 & $-100 \%$ \\
\hline Maximum (AU Provins [2018]) & 63,4 & 57,8 & $+102 \%$ \\
\hline Moyenne & 14,5 & 13,1 & $-12 \%$ \\
\hline
\end{tabular}




\begin{tabular}{|l|c|c|c|}
\hline Médiane & 12,2 & 11,7 & $-3 \%$ \\
\hline $1^{\text {er }}$ décile & 6,9 & 6,6 & $-100 \%$ \\
\hline $9^{\text {e }}$ décile & 26,0 & 20,0 & $+21 \%$ \\
\hline Écart-type & 9,2 & 7,7 & $38 \%$ \\
\hline Coefficient de variation & $63 \%$ & $59 \%$ & $317 \%$ \\
\hline $\begin{array}{l}\text { N. B. Les valeurs calculées pour les deux années sont produites à partir des aires équipées (au } \\
\text { moins un lit). }\end{array}$ \\
\hline
\end{tabular}

Sources : DREES, Statistique annuelle des établissements \& INSEE, recensement, exploitation des auteurs

L'analyse de l'évolution du coefficient de variation de la densité de lits tend à signaler une forme d'harmonisation des situations entre les villes: le coefficient de variation diminue de $63 \%$ à $59 \% .60$ villes réparties sur l'ensemble du territoire ont en effet vu leur densité de lits augmenter entre 1 et $102 \%$, parmi lesquelles Périgueux, Toulouse, Arras, Douai-Lens, Béthune, Saint-Étienne (Carte 1b). Ainsi au niveau des aires urbaines, l'évolution à la baisse du coefficient de variation indique lui aussi une réduction des disparités, une forme de convergence.

Carte $1 \mathrm{~b}$ - Taux d'évolution de la densité de lits de réanimation dans les aires urbaines équipées entre 2008 et 2018 et stock initial en 2008.

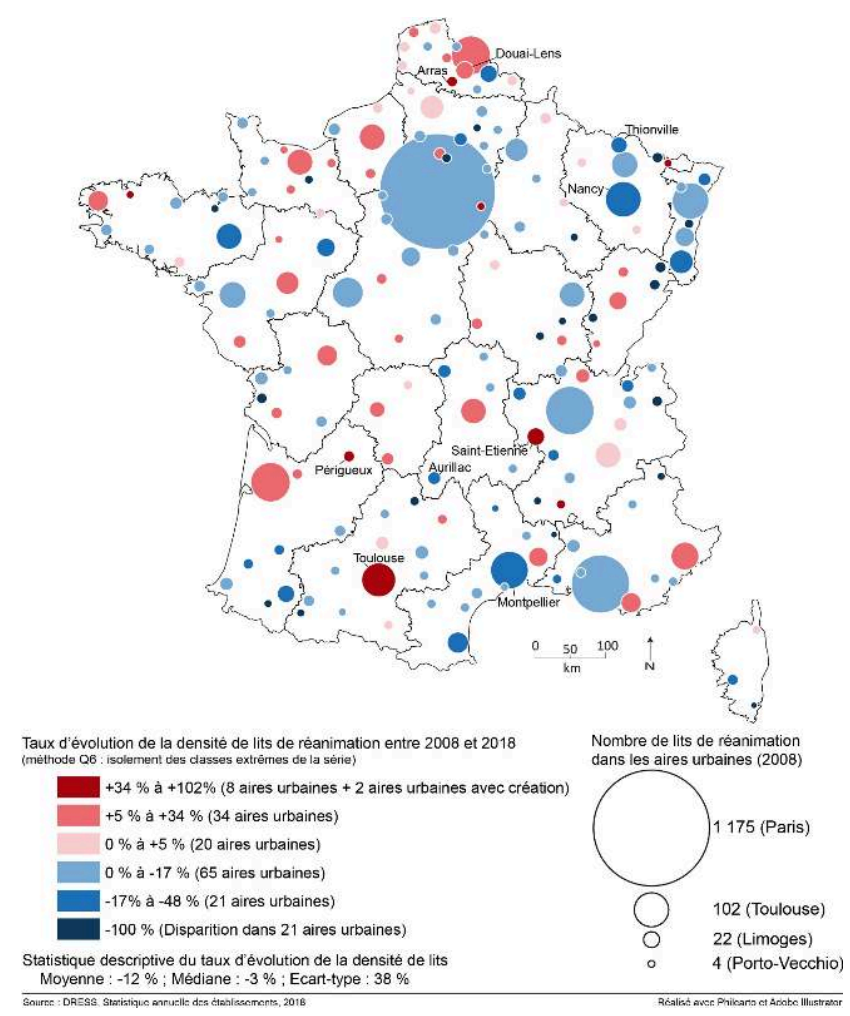

Sur la période, la convergence inter-régionale observée grâce à l'examen des paramètres de dispersion de la densité de lits s'accompagne-t-elle d'une forme de rattrapage par le bas dans les régions les plus équipées ? Une régression linéaire entre la densité initiale de lits en 2008 et le taux de variation du nombre de lits au cours de la période (2008-2018) permet de confirmer cette hypothèse. En effet, les régions avec les 
densités de lits de réanimation les plus faibles en 2008 sont celles qui ont connu des taux de variation du nombre de lits les plus positifs ; à l'inverse, les régions les mieux équipées en 2008 sont celles pour lesquelles les taux de variation sont les plus négatifs (Figure $1, \mathrm{R}^{2}=0,23$, coefficient de corrélation $=0,47$ [significatif à $5 \%$ ]). Toutefois, une grande partie $(77 \%)$ de cette variation régionale du taux d'évolution du nombre de lits reste inexpliquée par cette relation linéaire. Le cas de certaines régions est bien pris en compte par le modèle (Centre, Rhône-Alpes, Alsace), d'autres au contraire sont moins bien prises en compte (Corse, Picardie, Midi-Pyrénées, Aquitaine). D'autres facteurs doivent être convoqués: les évolutions démographiques, les stratégies des Agences Régionales de Santé pourraient apporter une amélioration du niveau d'explication.

Figure 1 - Relation entre la densité de lits de réanimation des régions en 2008 et le taux d'évolution des lits de réanimation des régions entre 2008 et 2018.

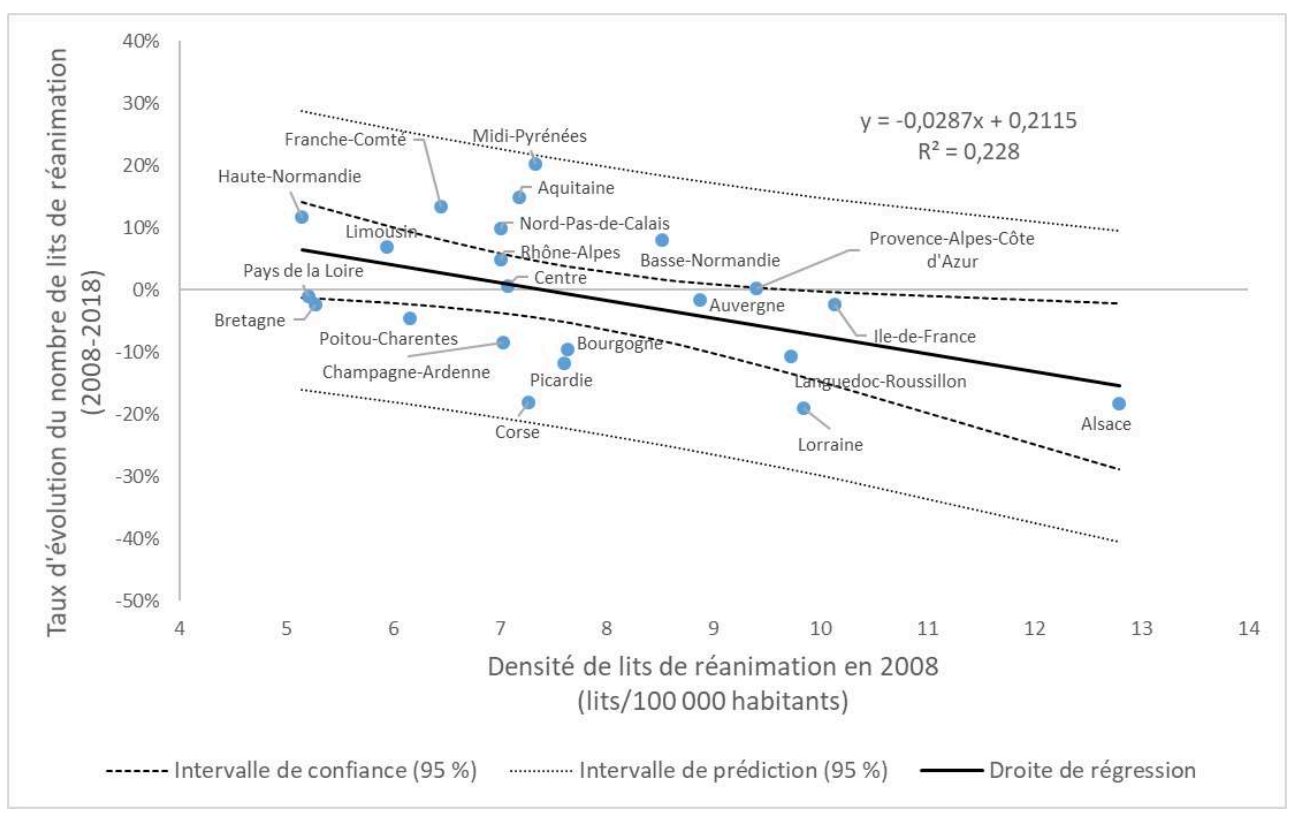

Si une convergence par le bas est observée entre régions, elle n'apparaît pas au niveau du système urbain: les tests de corrélations linéaires entre le taux d'évolution de la densité de lits et le niveau de densité en 2008 ne montrent pas de corrélation significative. Il n'existe pas non plus de relation linéaire entre le taux d'évolution de la densité de lits de réanimation et la taille des aires urbaines (nombre d'habitants de l'aire urbaine en 2008 ou 2016), ou la dynamique démographique (évolution de la population entre 2008 et 2016).

Depuis 10 ans, le niveau d'équipement en lits de réanimation a donc baissé relativement à l'augmentation de la population. Cette baisse s'est accompagnée d'une réduction des disparités interrégionales et d'une convergence vers un niveau médian de 7,1 lits pour 100000 habitants. La baisse généralisée des densités de lits n'est pas surprenante et s'inscrit de manière assez attendue dans la dynamique générale de l'équipement en lits hospitaliers et dans les trains de réformes qui ont présidé à l'évolution de l'équipement hospitalier depuis plus de trois décennies désormais. En dépit des cris d'alarme répétés des services d'urgence, des fédérations ou syndicats de médecins hospitaliers, la baisse de lits de court séjour est inexorablement conduite au nom de la modernisation du système de soins et de la productivité : cette dernière, mesurée en taux d'occupation des lits, en diminution des temps de séjours s'est faite 
sans envisager la possibilité que les situations de crises sanitaires puissent se prolonger. Les plans blancs, inscrits dans la loi hospitalière depuis 2004, permettent de répondre à l'urgence des crises sanitaires. Ils n'ont toutefois pas envisagé que ces situations de crises se prolongent ou se superposent à des situations déjà dénoncées de saturation de l'occupation des lits. Si la réduction des disparités inter-régionales de densités de lits de réanimation ne peut qu'être saluée, il faut aussi souligner que la baisse des densités a exercé, au début de la pandémie et au cours de la seconde vague, une pression telle sur les services de réanimation, que des mesures externes aux capacités hospitalières ont été mises en œuvre pour ralentir la progression de l'épidémie : confinement total ou partiel sont ainsi venus répondre à l'incapacité dans laquelle se sont trouvés les hôpitaux de répondre à l'augmentation brutale et prolongée du nombre de patients graves.

\section{Hypothèse 3 . En situation de crise pandémique, des inégalités dans le potentiel d'adaptation de l'équipement en lits}

Pendant le printemps 2020, en transformant les lits de soins intensifs ou de surveillance continue en lits de réanimation, les équipes soignantes ont été capables d'adapter les services de soins hospitaliers à la pénurie de lits de réanimation. Dans le temps court de la pandémie, comment ces transformations pouvaient-elles être opérées dans les régions et dans les villes équipées de lits de réanimation? Les régions et les villes possédaient-elles des ressources comparables en lits pour augmenter leur niveau d'équipement en lits de réanimation?

Tableau 3a - Paramètres de tendance centrale et de dispersion du nombre de lits de réanimation, de soins intensifs et de surveillance continue dans les régions en 2018

\begin{tabular}{|l|c|c|c|}
\hline & Réanimation & Soins intensifs & Surveillance continue \\
\hline Nombre de régions équipées & 22 & 22 & 22 \\
\hline Somme de lits & 4948 & 5686 & 7175 \\
\hline Minimum (Corse) & 18 & 21 & 22 \\
\hline Maximum (Ile-de-France) & 1153 & 1256 & 1212 \\
\hline Moyenne & 225 & 258 & 326 \\
\hline Médiane & 171 & 173 & 292 \\
\hline Ecart-type & 231 & 247 & 260 \\
\hline Coefficient de variation & $103 \%$ & $96 \%$ & $80 \%$ \\
\hline
\end{tabular}

Source : DREES, Statistique annuelle des établissements, exploitation des auteurs

Le nouvel indicateur proposé a été calculé au niveau des anciennes régions. Pour ce niveau géographique d'analyse, tous les lits de réanimation, surveillance continue et soins intensifs de France métropolitaine sont considérés, qu'ils soient situés dans une aire urbaine ou non. Ce choix n'a pas d'effet notable sur le total des lits de réanimation, car seuls 25 lits se trouvent en dehors d'une aire urbaine ${ }^{29}$. En revanche 
pour les lits de surveillance continue et de soins intensifs, la somme des lits de ce type est plus importante que pour le niveau des aires urbaines : il augmente de 11828 à 12 861 lits, soit une prise en compte d'environ 1000 lits supplémentaires (Tableau 3a).

$\mathrm{Au}$ niveau des régions, le potentiel de transformation en lits de réanimation, au niveau national, est donc de $260 \%{ }^{30}$ : ainsi il y a une possibilité théorique de multiplier par 3,6 le nombre de lits de réanimation. Le potentiel moyen des 22 anciennes régions est de $284 \%$ et le potentiel médian de transformation est de $271 \%$. Les différences interrégionales sont assez faibles : le coefficient de variation n'est que de $27 \%$ et l'écart-type de $76 \% .21$ des 22 régions ont un potentiel de transformation compris entre $181 \%$ et $359 \%$ (multiplication par 2,8 à 4,5). Le Limousin est un cas à part, avec un potentiel de transformation étonnamment important de $553 \%$ qui s'explique par la situation de Limoges où les ressources en lits de soins intensifs et de surveillance continue sont importantes eu égard au nombre de lits de réanimation (Potentiel de transformation de $683 \%$ ). La carte du potentiel de transformation des régions (Carte $2 \mathrm{a}^{31}$ ), souligne la situation de régions comme la Basse-Normandie, l'Auvergne, l'île-de-France à faible potentiel et celles de Bretagne, Pays de la Loire, Haute-Normandie, Bourgogne, Franche-Comté où le potentiel est fort. Les régions très touchées par l'épidémie au printemps 2020 ont toutes de faibles potentiels et ont été contraintes de déplacer des patients vers des régions plus épargnées par l'épidémie ${ }^{32}$.

Carte $2 a-$ Volume de lits de réanimation et potentiel de transformation des anciennes régions en 2018

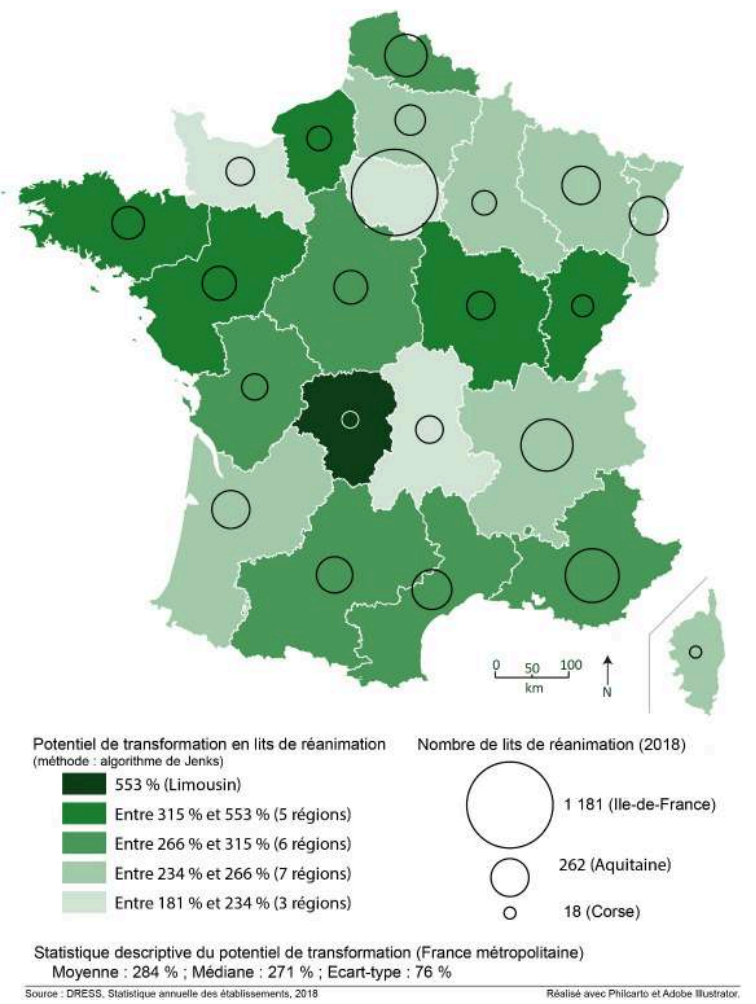

33 Au niveau des aires urbaines, en 2018, la France compte 5545 lits de soins intensifs et 6 283 lits de surveillance continue dans les 150 aires urbaines équipés d'au moins un lit de réanimation en 2018. Avec au moins 160 lits chacune, Paris et 14 métropoles ${ }^{33}$ regroupent $57 \%$ de ces deux catégories de lits (Tableau 3b). L'équilibre entre les trois catégories de lits, réanimation, soins intensifs, surveillance continue, varie fortement 
d'une ville à l'autre. Si Paris dispose d'un tiers de lits de chaque type, Nice offre $21 \%$ de lits de réanimation, $21 \%$ de lits de soins intensifs et $58 \%$ de lits de surveillance continue : ainsi à Nice, il faudrait transformer $80 \%$ des lits pour obtenir $100 \%$ de lits assimilables à des lits de réanimation, tandis qu'à Paris il faudrait en transformer "seulement" $66 \%$.

À court terme, le potentiel de transformation, basé sur la capacité de faire évoluer des lits de soins intensifs et de surveillance continue en lits de réanimation, est de $240 \%$ au niveau national pour les aires urbaines équipées. Cela signifie que le potentiel de transformation permettrait de multiplier par 3,4 le volume de lits de réanimation existant, soit le passage de 4923 lits de réanimation à 16751 lits potentiellement équipés en réanimation. Ce chiffre, très théorique, est bien supérieur aux 8000 lits du pic épidémique du printemps 2020, mais plus proche de l'objectif des 12000 lits de réanimation évoqués par le ministre de la Santé au cours de l'été $2020^{34}$. En moyenne, ce potentiel est de $240 \%$ pour les aires urbaines ${ }^{35}$. Localement il varie fortement (écarttype de $117 \%$ ), de $33 \%$ à Digne-les-Bains, ou $55 \%$ à Thonon-les-Bains jusqu'à $706 \%$ à Avignon ou 683 \% à Limoges.

Tableau $3 \mathrm{~b}$ - Paramètres de tendance centrale et de dispersion du nombre de lits de réanimation, de soins intensifs et de surveillance continue dans les 150 aires urbaines équipées en lits de réanimation en 2018

\begin{tabular}{|l|c|c|c|}
\hline & Réanimation & Soins intensifs & Surveillance continue \\
\hline Nombre d'AU équipées & 150 & 131 & 148 \\
\hline Somme de lits dans les AU équipées & 4923 & 5545 & 6283 \\
\hline Minimum & 3 & 4 & 2 \\
\hline Maximum (AU de Paris) & 1145 & 1256 & 1204 \\
\hline Moyenne & 33 & 42 & 42 \\
\hline Médiane & 12 & 16 & 16 \\
\hline $1^{\text {er décile }}$ & 8 & 6 & 4 \\
\hline $9^{\text {e décile }}$ & 64 & 86 & 109 \\
\hline Écart-type & 99 & 116 & $257 \%$ \\
\hline Coefficient de variation & $302 \%$ & $274 \%$ & \\
\hline
\end{tabular}

Source : DREES, Statistique annuelle des établissements, exploitation des auteurs

Les grandes aires urbaines ressortent avec des potentiels de transformation situés autour de la moyenne (Carte $2 \mathrm{~b})^{36}$. Dans certaines métropoles toutefois (Nantes, Rennes, Rouen) le potentiel de transformation est très important (supérieur à $300 \%$ ) : cela signifie aussi que la réserve en lits de soins intensifs et surveillance continue est élevée comparativement au nombre de lits de réanimation. Un faible lien de corrélation est observé entre la taille des aires urbaines et leur potentiel de transformation $\left(R^{2}=0,18\right.$ avec Paris, $R^{2}=0,21$ sans Paris). Toutefois aucun lien n'est observé entre le nombre de lits de réanimation dont disposent les aires urbaines en 2018 et ce potentiel : ce n'est pas parce qu'une aire urbaine possède un nombre important de lits de réanimation, qu'elle possède un potentiel important, ou inversement. 
Carte $2 b$ - Volume de lits de réanimation potentiel de transformation des aires urbaines équipées d'au moins un lit de réanimation en 2018

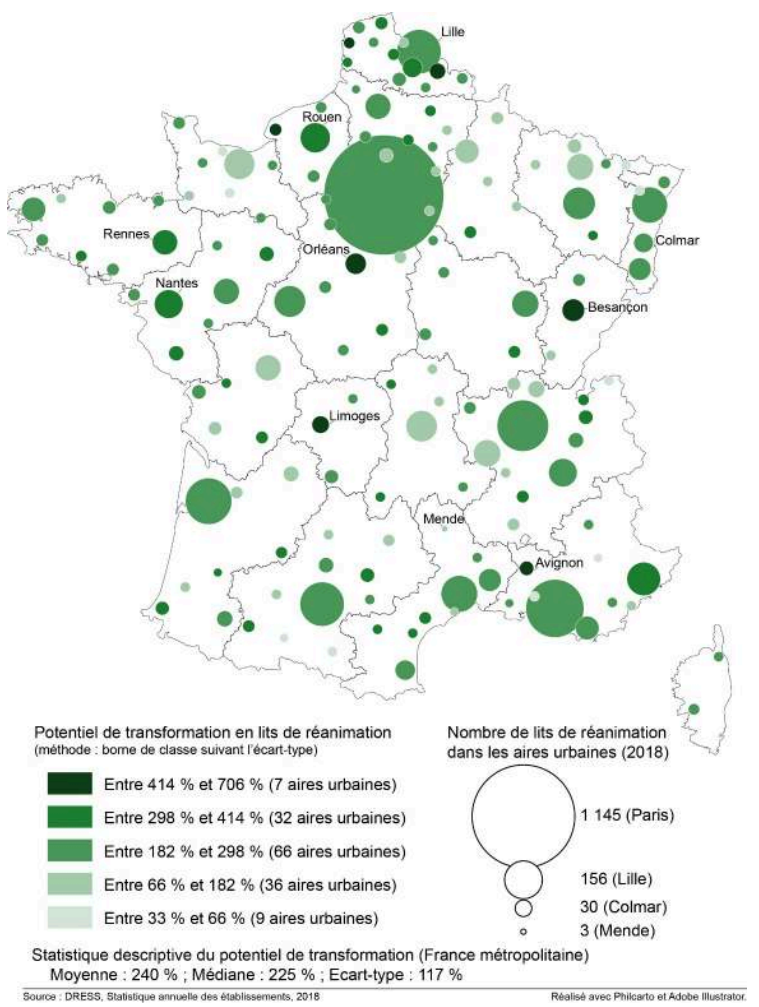

Enfin, nous avons testé l'existence d'une relation entre le potentiel de transformation et la densité de lits de réanimation en 2018 (Figure 2) et observé une corrélation négative relativement élevée $\left(\mathrm{R}^{2}=0,38\right.$, coefficient de corrélation $=0,62$ [significatif à $1 \%]$ ). Les régions avec une faible densité de lits de réanimation en 2018 ont un potentiel de transformation plus important ${ }^{37}$ que les régions avec des densités de lits de réanimation élevées ${ }^{38}$. Ainsi le potentiel de transformation pourrait suppléer à la faible densité de lits de réanimation. Ces observations signifient-elles que les ARS adoptent des stratégies différentes dans leur offre de soins d'urgence? Difficile de le dire à travers l'utilisation de ces seuls indicateurs. Toutefois, ces résultats éclairent sur les ressources, sur les potentiels de transformation en seuls termes d'équipement. Mais audelà de l'équipement en lits c'est bien sûr la présence d'un nombre de soignant.es suffisant qui est primordiale pour répondre à une pandémie dont le terme n'est pas encore connu. 
Figure 2 - Relation entre le potentiel de transformation régionale de lits de réanimation et la densité de lits de réanimation de chaque région en 2018 (hors Limousin)

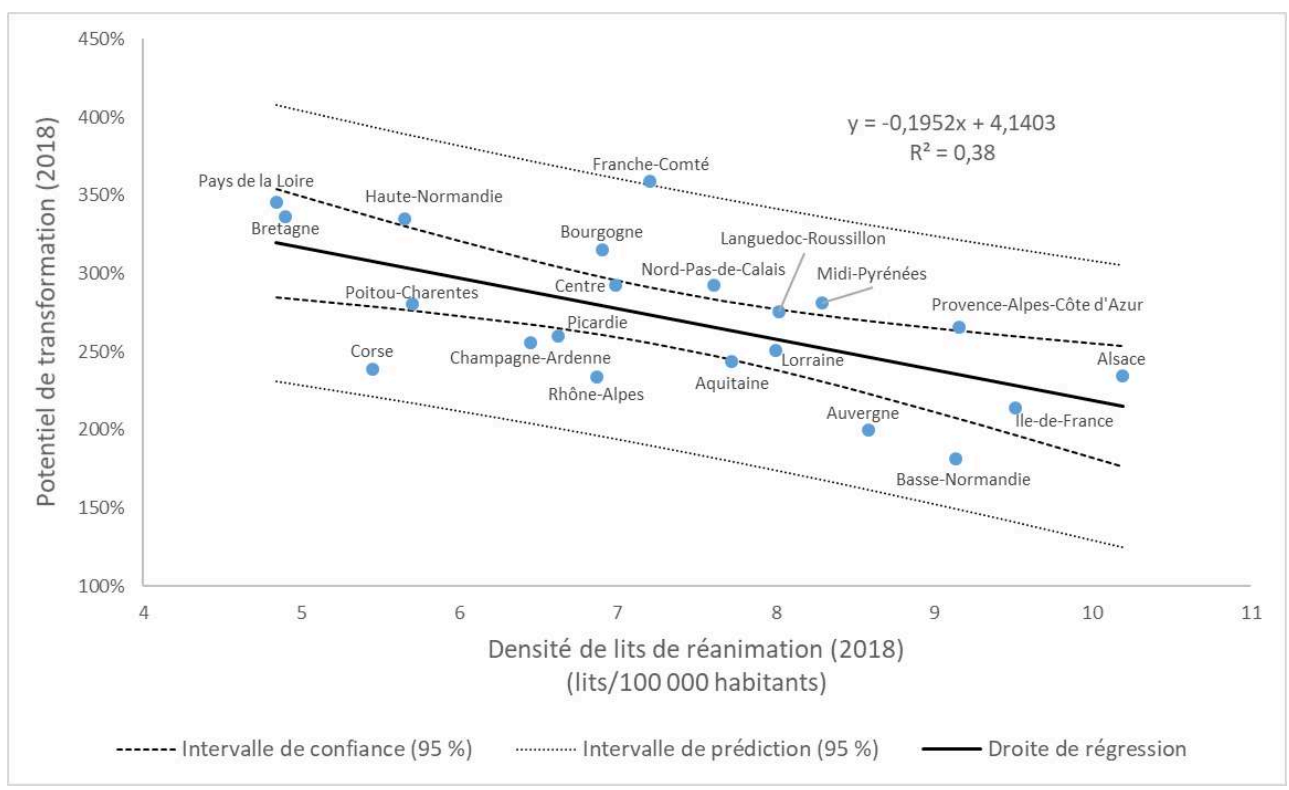

\section{Discussion}

Si l'évolution de la géographie de l'équipement hospitalier est bien documentée en France, le cas particulier de l'équipement de réanimation, très sélectif puisque seules 150 villes en sont équipées, l'est moins. Que ce soit au niveau des aires urbaines ou des régions, des changements importants se sont produits depuis une dizaine d'années dans la géographie des lits de réanimation. En situation de réforme hospitalière, et contrairement aux lits de courts séjours qui ont accusé une baisse importante depuis les années 2000 (36048 lits supprimés, dont 33935 en chirurgie), le volume de lits de réanimation est resté relativement stable. Toutefois cette apparente stabilité masque des transformations spatiales importantes : une cinquantaine de villes a gagné des lits, mais une soixantaine en ont perdu dont 21 aires urbaines où ils ont complètement disparu. Par ailleurs 107 des 150 villes initialement équipées ont connu une baisse de densité de lits par habitant. Contrairement à l'ensemble des lits de court séjour ou aux maternités, les tests statistiques n'ont pas permis, au niveau national, d'expliquer ces changements par la taille des villes ou par le niveau d'équipement initial. Certes, les 21 aires urbaines qui ont perdu leur équipement de lits de réanimation sont plutôt des petites aires urbaines, mais aucune relation linéaire entre le taux d'évolution de la densité de lits et le nombre d'habitants ou le taux d'évolution de la population des aires urbaines n'est observée. Si le volume de lits ne diminue pas, le niveau d'équipement, mesuré en densité de lits par habitant baisse. Cette baisse s'inscrit dans un mouvement général de réduction observé pour l'ensemble des soins hospitaliers dans les pays de l'OCDE (Vas et al., 2008). Elle s'explique par la diffusion d'une approche gestionnaire des soins qui vise à augmenter la productivité hospitalière en réduisant les temps de séjour et les capacités en hospitalisation de courte durée (Belorgey \& Pierru, 2017). Celle-ci a été encouragée par l'utilisation des outils du New Public Management. En France pour les lits de courts séjours (Conti et al., 2020), en Nouvelle-Zélande (Barnett, 1999), en Italie (De Belvis et al., 2012), au Canada (Carrière et al., 2020 ; Liu et al., 2001), 
la réduction des équipements hospitaliers a été de pair avec une amplification des inégalités territoriales. Dans le cas des lits de réanimation c'est le même constat concernant la réduction du nombre de villes équipées en lits, ou l'abaissement des densités de lits au niveau des villes. En considérant seulement les villes équipées, une réduction des disparités de densités de lits par habitant accompagne la baisse générale. $\mathrm{Au}$ niveau régional aussi, la réduction des inégalités accompagne une baisse des densités de lits par habitant. Ce résultat s'accorde avec des travaux qui ont mis en évidence une convergence inter-régionale des lits de courts séjours (Lucas-Gabrielli \& Tonnellier, 2000 ; Baudet-Michel et al., 2019) ou des lits par accouchements (Baillot \& Evain, 2013). Néanmoins, la convergence s'effectue par le bas: les régions les mieux équipées en 2008 ont connu une baisse de leur volume de lits de réanimation; les régions les moins denses une augmentation.

En situation de crise sanitaire, et de pandémie de Covid-19, les potentiels des équipes soignantes pour adapter l'équipement insuffisant en lits de réanimation ont inspiré l'indicateur inédit de potentiel de transformation des lits de soins intensifs et de surveillance continue en lits de réanimation. Son résultat au niveau national (possibilité de multiplier par 3,4 le volume de cet équipement) est bien supérieur aux objectifs fixés par le gouvernement pour faire face à une deuxième vague : 12000 lits, soit une multiplication par 2,4. Plus ce potentiel de transformation est élevé, plus l'effort de transformation à opérer est important. D'autant plus dans un contexte où les équipes soignant.es semblent exsangues. Le potentiel théorique calculé pour cet article souligne aussi les inégalités entre régions et villes dans leur capacité à augmenter quantitativement le volume de lits de réanimation. Certaines villes pouvant multiplier leur volume jusqu'à 6 à 8 tandis que d'autres peuvent à peine doubler leur capacité. À titre d'exemple, 470 lits de réanimation équipaient la région Grand Est au début de l'année 2020. Ce nombre a été porté à plus de 1200 au plus fort de la crise avec notamment l'installation d'un hôpital militaire de campagne (Eichwald \& Moreau, 2020). Au niveau régional il faut aussi souligner que le potentiel de transformation semble suppléer à la faible densité des lits en 2018.

Ces résultats posent plus globalement la question de la possibilité pour les hôpitaux de faire face à des crises épidémiques qui ne sont malheureusement pas nouvelles. Si la crise de la Covid-19 est exceptionnelle par son ampleur, en 2009-2010, la pandémie de H1N1 avait engendré un pic d'admission en décembre 2009 et les séjours hospitaliers pour grippe (14680) avaient été multipliés par cinq par rapport aux années 2007-2008, les séjours en réanimation ou unité de soins intensifs par 10 (1748) (Bonmarin \& LevyBruhl, 2012). La Covid-19 n'est pas la première crise à laquelle le système hospitalier doit faire face. Elle survient après 20 ans de réformes techniques qui ont certes amélioré la qualité de certains soins et ont permis le développement du secteur ambulatoire à l'hôpital, mais aussi de réformes managériales qui ont mis l'hôpital, et particulièrement l'hôpital public sous une pression gestionnaire qui paraît insoutenable dans le cadre d'une crise sanitaire prolongée.

\section{Conclusion}

Nos résultats permettent de montrer que dans la situation de réforme chronique que vivent les hôpitaux, certaines régions comme la Lorraine, l'île-de-France, et villes (Paris, Metz ou Marseille) ont enregistré une diminution de leur équipement en lits de 
réanimation au cours des 10 dernières années. Au printemps 2020, ces régions et villes ont été particulièrement en tension pour répondre à l'augmentation du flux de patients. Par ailleurs, si le nombre de lits n'a globalement pas baissé à l'échelle de la France entière (5000 environ), le nombre d'habitants a augmenté, si bien que les densités de lits par habitant ont partout baissé : autant au niveau régional, de 7,7 à 7,3 en moyenne, qu'au niveau des villes de 14,5 à 13,1 (lits/100 000 habitants). Cette baisse s'est accompagnée d'une réduction des disparités interurbaines et interrégionales, et d'une convergence au niveau régional: les régions les mieux équipées en 2008 sont celles qui en ont le plus perdu. En période de crise sanitaire, les personnels des hôpitaux ont fait la preuve de leur capacité à faire face dans l'urgence, notamment à travers la transformation d'un certain nombre de lits de soins intensifs et de surveillance continue en lits de réanimation. Leurs totaux respectifs sont de 5686 et 7175 lits en France en 2018, ce qui permet théoriquement de multiplier les capacités d'accueil par 3,6 au niveau national, pour monter potentiellement à 16751 lits. Toutefois, les capacités de transformations varient fortement selon les régions et selon les aires urbaines et ces capacités de transformation ne représentent rien si les personnels soignants ne sont pas là pour fournir concrètement les soins et la surveillance nécessaire pour les malades les plus atteints. Les soignant.es ont appris de la première vague épidémiques et les services se réorganisent dans le cadre des "Plan Blanc' au moment où les flux entrants augmentent drastiquement. Ainsi les personnels médicaux des services spécialisés (par exemple de médecine interne) sont temporairement affectés aux services de réanimation ou aux unités COVID. Ainsi, les personnels soignants, qui étaient déjà sous la pression des réformes conduites depuis plus de deux décennies, sont encore plus éprouvés par les tensions engendrées par une pandémie qui dure.

41 Alors que la France et de nombreux pays européens se trouvent au cœur d'une deuxième vague épidémique débutée à l'automne 2020, de nouvelles mesures limitant la liberté de se déplacer (confinement ou couvre-feu) sont mises en place afin de limiter la pression sur les services de réanimation. Car au-delà des autres critères mettant en lumière l'aggravation de la situation sanitaire (par exemple le taux d'incidence), c'est à nouveau la crainte de voir les hôpitaux saturés qui inquiète le plus. Ne pas avoir à choisir entre des patients atteints de la Covid-19 et d'autres patients, ne pas avoir à exercer dans des conditions proches des interventions militaires d'urgence, ne pas avoir à répondre à l'urgence par des transferts porteurs de dissémination de l'épidémie, voilà qui inscrit les politiques de réduction d'équipement des trois dernières décennies dans une perspective d'équité territoriale. Si les transferts de patients ont contribué à contenir la tension des services de réanimation des régions très affectées au printemps 2020, qu'en sera-t-il dans un contexte où l'ensemble des régions sera affecté de façon équivalente? Les tensions mises à jour par la pandémie conduiront-elles les pouvoirs publics à revoir les stratégies de concentration et de fermetures mises en œuvre depuis plus de 30 ans? Les revendications des soignant.es pour davantage de moyens humains, d'équipement seront-elles entendues? 


\section{BIBLIOGRAPHIE}

Andréo C., 2004, "La gestion des flux de malades dans les services de réanimation". Sociétés contemporaines, $\mathrm{n}^{\circ} 2$, p. 99-124.

Baillot A., Evain, F., 2013, "Les maternités : un temps d'accès stable malgré les fermetures". Journal de gestion et d'économie médicales, vol. 31, nº 6, p. 333-347.

Barnett J.R., 1999, "Hollowing out the state? Some observations on the restructuring of hospital services in New Zealand". Area, 31, 1, pp. 259-270.

Barczak A., Hilal M., 2016, "L'accès aux commerces et services dans les territoires de vie du quotidien". In Blancard S. et al. (dir), Campagnes du quotidien. Editions QAE "update Sciences \& Technologies", pp. 97-116.

Baudet-Michel S., Conti B., Chouraqui J., Commenges H., Delage M., Guérois M., Guiraud N., Le Neindre C., Madry P., Paulus F., Pavard A., Toutin G., Vallée J., Fol S., Quéva C., 2019, La rétraction des services et commerces dans les villes petites et moyennes : modalités et logiques à l'échelle interurbaine. Rapport CGET-CDC, $208 \mathrm{p}$.

Belorgey N., 2010, L'hôpital sous pression, enquête sur le nouveau management public. Paris, La Découverte, 336 p.

Belorgey N., Pierru F., 2017, "Une consultocratie hospitalière ? Les consultants, courtiers de la réforme du système de santé". Les tribunes de la santé, n 55/2, pp. 45-57.

De Belvis A. G., Ferrè F., Specchia M. L., Valerio L., Fattore G., Ricciardi W., 2012, "The financial crisis in Italy: implications for the healthcare sector". Health policy, vol. 106, n 1, p. 10-16.

Bonmarin I., Levy-Bruhl D., 2012, Analyse des données d'hospitalisation en France à partir du PMSI pendant la période pandémique 2009/2010. Saint-Maurice InVS : 78, tabl., fig. (Source : 1 hopital-en-france.pdf IRDES 2019)

Carpentier D., Beduneau G., Girault, C., 2015, "Séjour prolongé en réanimation". Réanimation, vol. $24, \mathrm{n}^{\circ} 4$, p. 379-388.

Carriere, K.C., Roos, L.L., Dover, D.C., 2000, "Across time and space: variations in hospital use during Canadian health reform". Health Services Research, 35, pp.467-487.

Conti B., Baudet-Michel S., Le Neindre C., 2020, "Envisager la rétraction d'un équipement dans le système urbain français : le cas des lits d'hospitalisation en court séjour". Géographie, économie, société, vol. 22, n 1, p. 5-33.

Delas A., 2011, "L'hôpital public, un nouvel acteur territorial entre aménagement sanitaire et rivalités stratégiques". Hérodote, vol. 4, p. 89-119.

Domin J. P., 2015, "Réformer l'hôpital comme une entreprise. Les errements de trente ans de politique hospitalière (1983-2013)". Revue de la régulation. Capitalisme, institutions, pouvoirs, $n^{\circ} 17$.

Dubas-Jakóbczyk K., Albreht T., Behmane D., Bryndova L., Dimova A., Džakula A., Habicht T., Murauskiene L., Scîntee S.G., Smatana M., Velkey Z., Quentin W, 2020, "Hospital reforms in 11 Central and Eastern European countries between 2008 and 2019: A comparative analysis". Health Policy, vol. 124, n 4, p. 368-379. 
Eichwald A., Moreau S., 2020, "Une sollicitation exceptionnelle des services de réanimation du Grand Est pendant la période de confinement". INSEE Analyses Grand Est, n 117, 4 p.

Franzini L., Giannoni M., 2010, "Determinants of health disparities between Italian regions". BMC Public Health, vol. 10, $\mathrm{n}^{\circ}$ 1, p. 296.

Gandré C. \& Or Z., 2020, France: Physical infrastructure, Germany: Physical infrastructure, in COVID-19 Health System Monitor, 10.7.2020, World Health Organisation. https:// www.covid19healthsystem.org/countries/france/livinghit.aspx? Section $=2.2 \% 20$ Workforce\&Type $=$ Chapter

Granger B., Pierru F., 2012, L'hôpital en sursis. Idées reçues sur le système hospitalier. Paris, Le Cavalier Bleu, $182 \mathrm{p}$.

Jousseaume V., 2002, "Diffusion et recomposition de l'offre médicale : l'exemple des Pays de la Loire (1967-2000)". in Fleuret S., Séchet R. (dir.), La Santé, les soins, les territoires. Penser le bienêtre. Rennes, Presses universitaires de Rennes, Collection Espace et territoires, p. 33-49.

Liu L., Hader J., Brossart B., White R., Lewis S., 2001, "Impact of rural hospital closures in Saskatchewan, Canada". Social Science \& Medicine, vol. 52, n 12, p. 1793-1804.

Lucas-Gabrielli V., Tonnellier F., 2000, "Les équipements hospitaliers en France". In Mattei M.-F., Pumain D. (coord.), Données urbaines 3. Paris, Anthropos-Economica, Collection Villes, pp. 189-198.

OCDE, 2017, OECD, Health Statistics 2017, OECD Publishing, Paris.

Perucca G., Piacenza M., Turati G., 2019, "Spatial inequality in access to healthcare: evidence from an Italian Alpine region". Regional Studies, 53, pp.478-489.

Tritsch L., Rohmer D., Diebold L., Simon M., Frantz M., Haubtmann T., Krebs Y., Stock N., Benhassine E., 2020, "Transferts hélioportés interhospitaliers de patients atteints de COVID-19 : expérience du SDIS du Bas-Rhin". Médecine de catastrophe - Urgences collectives, vol. 4, $\mathrm{n}^{\circ} 3$, pp. 181-186.

Vas G., Sebestyen A., Agoston I., Varga S. Kriszbacher I., Belehem J., Boncz I., et al., 2008, "Comparative analysis of Hospital Bed capacities of old (EU-15) and new (EU-12) memberstates of the European Union". Value in Health, vol. 11, Issue 6.

Vigneron E., Haas S., 2009, 10 ans de recomposition hospitalière. Paris, Dexia, 16 p.

Vigneron E., 2017, L'Hôpital et le territoire. Paris, Techniques Hospitalières, SPH Editions, 295p.

\section{ANNEXES}

\section{Construction d'une base longitudinale des lits de réanimations au niveau des aires urbaines (zonage 2010) de 2008 à 2018}

Afin de pouvoir analyser l'évolution de la présence des lits de réanimation dans les territoires français, deux années de la SAE ont particulièrement été mobilisées : 2008 et 2018. Or pour ces deux années la méthode de requêtes des lits de réanimation a évolué sur deux points :

- (i) d'une part le bordereau consacré à la « Réanimation, surveillance continue et soins intensifs » a évolué 
- (ii) et d'autre part les entités enquêtées ont évolué.

- (i) Concernant le premier point, le changement dans le mode d'enquête de la SAE concernant les lits de réanimation repose sur un regroupement des informations sur un seul et même bordereau alors que ces informations étaient auparavant renseignées par les établissements dans quatre bordereaux distincts. En effet, une précision du document d'aide au remplissage de la SAE de 2013, année de changement de l'enquête, le précise :

CORRESPONDANCE AVEC LA SAE 2012

L'activité de ces différentes unités était décrite dans les bordereaux Q14A pour les enfants et Q14B pour les adultes. Les unités liées à la chirurgie cardiaque ou interventionnelle, aux USIC ou la neurochirurgie figurait dans les bordereaux Q11, Q12, et les soins intensifs dans l'Unité Neurovasculaire dans le Q08.

(Aide au remplissage, SAE 2013, p.129)

Concernant les lits de réanimation, la différence notable entre l'année 2008 et 2018

des données tient dans le cas particulier des lits de réanimations de chirurgie cardiaque.

- En effet, en 2008, l'aide au remplissage du bordereau Q14B (adulte), intitulé « Réanimation, soins intensifs spécialisés et surveillance continue », indique que «Les unités liées au traitement de grands brûlés, à la chirurgie cardiaque ou la neurochirurgie comme la réanimation néonatale et les soins intensifs de néonatologie ne doivent pas figurer dans ce bordereau » (p.119).

- En 2018, l'aide au remplissage du bordereau «REA » indique quant à lui : «la réanimation et les soins intensifs de néonatologie qui, faisant partie intégrante de l'autorisation octroyée en périnatalité, est à ce titre décrite dans le bordereau correspondant, et celle des grands brûlés, également indissociable de l'autorisation d'activité et par conséquent décrite dans le bordereau particulier dédié à cette prise en charge » (p.147). Ainsi, les soins liés aux grands brûlés et à la néonatalogie continue d'être traités à part tandis que pour la neurochirurgie seuls les lits de soins intensifs des unités neurovasculaires (UNV) sont à prendre en compte dans ce bordereau, le reste étant décrit dans un autre bordereau spécifique : NEUROCHIR « Neurochirurgie et activités interventionnelles par voie endovasculaire en neuroradiologie ».

Afin d'assurer une comparaison entre l'année 2018 et 2008, les lits de réanimation du bordereau Q14B (Réanimation adulte) et du bordereau Q11 (Chirurgie cardiaque) ont été additionnés. Le tableau suivant rend compte de l'évolution pour chaque année depuis 2008).

Pour information concernant les variables utilisées pour calculer le nombre de lits de réanimation des trois bordereaux Q14B, Q11 et REA :

- Q14B : le nombre de lits est calculé à partir d'une sélection de la variable " UNITE » : seules les unités de réanimation sont sélectionnées (codées « R1 », « $\mathrm{R}^{2}$ », «R3», «R4 » et « R5 »).

- Q11 : le nombre de lits est calculé à partir d'une sélection de la variable « CCARD » (Chirurgie cardiaque) : seules les unités de réanimation de chirurgie cardiaque sont sélectionnées (codées « 2REA »).

- REA : le nombre de lits est calculé à partir d'une sélection de la variable " UNI » : seules les unités de réanimation adulte sont sélectionnées (codées « REAADU »). 
Tableau 4 - Nombre de lits de réanimation des bordereaux Q14B et Q11 pour les années 2008 à 2012 et du bordereau REA pour les années 2013 à 2018.

\begin{tabular}{|c|c|c|c|c|}
\hline AN & Q14B (Réa) & Q11 (Chir) & Q14+Q11 & REA \\
\hline 2008 & 4573 & 465 & 5038 & \\
\hline 2009 & 4573 & 461 & 5036 & \\
\hline 2010 & 4673 & 508 & 5181 & \\
\hline 2011 & 4530 & 442 & 4972 & \\
\hline 2012 & 4578 & $447^{*}$ & 5025 & \\
\hline 2013 & & & & 5024 \\
\hline 2014 & & & & 5032 \\
\hline 2015 & & & & 5026 \\
\hline 2016 & & & & 5087 \\
\hline 2017 & & & & 5050 \\
\hline 2018 & & & & \\
\hline
\end{tabular}

Sources : SAE de 2008 à 2018, France entière

* Pour l'année 2012 le bordereau Q11 des tables statistiques n'est pas correct, car les données présentent correspondent à celle du bordereau Q12. Pour pallier ce problème, nous avons exceptionnellement, dans une mesure de comparabilité des données, utilisé la table administrative de la SAE pour le bordereau Q11 (table non redressée par la Drees).

Une nouvelle table ad hoc a donc été réalisée afin d'additionner les lits des bordereaux Q14B et Q11 pour l'année 2008 au niveau de chaque hôpital.

-(ii) Concernant le second point, ce changement n'a pas eu d'impact pour notre travail sur les lits de réanimation, car toutes les entités géographiques étaient concernées pour remplir les bordereaux dès 2008 :

Pour le secteur public: bordereau à remplir au niveau de l'entité juridique monoétablissement, et au niveau de l'établissement géographique interrogé pour les entités juridiques multi-établissements. Pour le secteur privé : bordereau à remplir au niveau de l'établissement géographique.

(Aide au remplissage, SAE 2008, p.86 pour le bordereau Q08, p.103 pour le bordereau Q11, p. 107 pour le bordereau Q12, p.119 pour le bordereau Q14)

\section{NOTES}

1. Source: https://www.data.gouv.fr/fr/reuses/tableau-de-bord-de-suivi-delepidemie-de-coronavirus-en-france/, consulté le 2 janvier 2021.

2. "Les soins de réanimation sont destinés à des patients qui présentent ou sont susceptibles de présenter plusieurs défaillances viscérales aiguës mettant directement en jeu le pronostic vital et impliquant le recours à des méthodes de suppléance.» (Article R6123-33 du Code de Santé Publique).

3. «Les soins intensifs sont pratiqués [...] pour prendre en charge des patients qui présentent ou qui sont susceptibles de présenter une défaillance aigüe de l'organe concerné par la spécialité au titre de laquelle ils sont traités. » (Article D.6124-104 du Code de Santé Publique). 
4. "La surveillance continue est [...] organisée pour prendre en charge des malades qui nécessitent, en raison de leur état ou du traitement qui leur est appliqué, une observation clinique et biologique répétée et méthodique.» (Article D.6124-117 du Code de Santé Publique).

5. Organisation de coopération et de développement économiques.

6. En 2009, la loi HPST remplace les ARH par les ARS, qui deviennent ainsi aussi responsables de l'organisation des relations entre le secteur ambulatoire, l'Assurance Maladie et les établissements de soins.

7. En médecine, chirurgie, obstétrique, hospitalisation à domicile, urgence, imagerie médicale, psychiatrie, traitement du cancer, etc.

8. L'Arrêté ministériel du 5 mai 1992 propose un seuil minimal de 200000 habitants avec des indices de besoins pour découper l'espace régional en secteurs sanitaires. Des écarts peuvent exister: ainsi, en Bretagne, le secteur 5 de Rennes-Fougères-VitréRedon compte 800000 habitants et le secteur 8 de Loudéac-Pontivy n'en compte que 150000 (Lerouvillois \& Vinclet, 2002).

9. Des outils de gestion comme la Dotation Globale de Fonctionnement, laTarification à l'Activité, dite T2A, l'Objectif National des Dépenses d'Assurance Maladie ont pour ambition de contrôler et si possible réduire les dépenses d'hospitalisation et de soins pour l'Assurance Maladie.

10. Le NPM apparaît à la fin des années 1960 dans différents États de l'OCDE, dont les USA, la Grande-Bretagne, l'Australie, la Nouvelle-Zélande. Considéré par Pollitt et Bouckaert (2011) comme une doctrine, le NMP prône un accroissement de la performance, une réduction de la dépense publique, l'introduction de mécanismes de marché dans le secteur public (Bezes, 2009).

11. Source : https://www.oecd-ilibrary.org/sites/health_glance-2017-en/1/2/9/3/ index.html?itemId=/content/publication/health_glance-2017-

en\&_csp_=a2ae54f57e835a070fed1d8fd9a56f53\&itemIGO=oecd\&itemContentType=book\#sect-89, consulté le 2 janvier 2021.

12. Les lits de court séjour concernent les hospitalisations dans les établissements hospitaliers, hors des soins de suite et réadaptation (séjours de convalescence) et des soins longue durée (pour des pathologies longues). Le court séjour dure plus de $24 \mathrm{~h}$ et généralement moins de 8 jours.

13. https://www.fhf.fr/Offre-de-soins-Qualite/Organisation-de-l-offre-de-soins/ Covid-19-Besoins-en-reanimation-et-reponse-capacitaire-Recommandations-aux-

etablissements-de-sante, site de la fédération hospitalière de France, consulté le 10 novembre 2020.

14. https://www.lemonde.fr/les-decodeurs/article/2020/05/05/coronavirus-agemortalite-departements-pays-suivez-l-evolution-de-l-epidemie-en-cartes-etgraphiques_6038751_4355770.html, par lemonde.fr, consulté le 2 janvier 2021. https:// www.francetvinfo.fr/sante/maladie/coronavirus/infographies-Covid-19-mortshospitalisations-age-malades-l-evolution-de-l-epidemie-en-france-et-dans-le-mondeen-cartes-et-graphiques.html, par francetvinfo.fr, consulté le 2 janvier 2021.

15. https://www.leparisien.fr/paris-75/a-l-hopital-bichat-douze-semaines-de-luttecontre-le-coronavirus-et-ca-va-durer-tres-longtemps-16-04-2020-8300868.php, par leparisien.fr 
16. Pour cet article le terme de ville est parfois utilisé en lieu et place du terme d'aire urbaine. Dans tous les cas, il s'agit bien du périmètre des aires urbaines auquel le mot ville fait référence.

17. Site de téléchargement des bases: http://www.data.drees.sante.gouv.fr/ ReportFolders/reportFolders.aspx, consulté en avril 2020.

18. En téléchargement pour les fichiers spécifiques (bordereaux) qui détaillent la présence de cet équipement.

19. Site de l'Insee : https://www.insee.fr/fr/metadonnees/source/serie/s1321, consulté en janvier 2021.

20. Ce potentiel a été évalué au niveau des aires urbaines. Ainsi les lits de soins intensifs ou de surveillance continue d'hôpitaux non équipés en lits de réanimation, mais présents dans une aire urbaine ont tout de même été comptabilisés. C'est le cas dans les métropoles.

21. Au niveau de l'échantillon des aires urbaines, le solde entre 2008 et 2028 est de 60 lits. Pour l'ensemble des régions, 35 lits seulement ont été supprimés entre 2008 et 2018. La différence de 25 lits entre le niveau des aires urbaines et celui des régions repose sur la création d'un hôpital situé dans une commune rurale, en dehors d'une aire urbaine, entre Belfort et Montbéliard. Ces 25 sont comptés au niveau régional, mais ne sont pas comptés au niveau de l'échantillon des aires urbaines.

22. Elle est suivie de PACA (460 lits) et Rhône-Alpes (450 lits).

23. Mais aussi la Franche-Comté ( 85 lits) ou la Champagne-Ardenne (86 lits)

24. Deux aires urbaines qui n'étaient pas équipées en 2008 le sont en 2018 : Saint-Avold (36 000 habitants) et Digne-les-Bains (25 000 habitants).

25. 3 d'entre elles ont entre 50000 et 100000 habitants et les quinze autres entre 10000 et 50000 .

26. Marseille (-31 lits), Paris (-30 lits), Nancy (-30 lits), Montpellier (-20 lits), Strasbourg (-16 lits) ou encore Le Mans (-14 lits)

27. Toulouse ( +53 lits), Bordeaux ( +34 lits), Saint-Étienne (+30 lits), Lille (+23 lits), Nice (+20 lits), Clermont-Ferrand (+19 lits) ou encore Caen ( +15 lits) ou Poitiers (+14 lits)

28. $-18 \%$ en Languedoc-Roussillon, $-13 \%$ en Picardie et $-10 \%$ en Bourgogne

29. Ces 25 lits se trouvent dans l'hôpital Nord de Franche-Comté (Site Trévenans), situé dans la commune de Trévénans (1 200 habitants), issu de la fusion des hôpitaux de Belfort et Montbéliard.

30. Détail du calcul : $\mathrm{Pt}=([5686+7$ 175]/4 948)*100

31. L'algorithme de Jenks a été utilisé pour discrétiser les valeurs de potentiel de transformation associées à chacune des régions. Cette méthode fait ressortir les seuils naturels et permet de créer des classes homogènes. L'algorithme vise à trouver le nombre de classes souhaitées en minimisant la variance intra-classe et en maximisant la variance inter-classe. Source : http://magrit.cnrs.fr/docs/discretisation_fr.html

32. Source: https://dashboard.covid19.data.gouv.fr/transferts-de-patients? location=FRA

33. 558 lits à Marseille Aix-en-Provence, 451 lits à Toulouse, 446 à Lyon, 404 à Lille, 338 lits à Nice et 321 à Bordeaux (lits de soins intensifs et lits de surveillance continue additionnés) 
34. https://www.lemonde.fr/planete/article/2020/06/25/olivier-veran-nous-mettonstout-en-uvre-pour-eviter-de-devoir-un-jour-reconfiner_6044093_3244.html, consulté le 20 octobre 2020.

35. La médiane est de $225 \%$ et la courbe de distribution de cet indicateur suit une loi normale : une soixantaine de villes ont un potentiel compris entre $200 \%$ (triplement de l'offre) et $300 \%$ (quadruplement).

36. $215 \%$ à Paris, 212 \% à Lyon, 209 \% à Marseille, 291 \% à Toulouse, $259 \%$ à Lille, 188 \% à Bordeaux, $229 \%$ à Strasbourg ou encore $212 \%$ à Grenoble

37. Par exemple, la région Pays de la Loire avec la plus faible densité régionale de lits en 2018 (4,8 lits pour 100000 habitants) a un potentiel de transformation de $345 \%$. Le cas de la Bretagne est proche (4,9 lits/100 000 habitants) et un potentiel de $336 \%$.

38. L'Alsace est la région qui possède la densité de lits de réanimation la plus forte de France : 102 lits de réanimation pour 100000 habitants. En Île-de-France la situation est comparable: densité de lits importante de 9,5 lits/100 000 habitants et potentiel de transformation faible de $214 \%$.

\section{RÉSUMÉS}

Les lits de réanimation ont été particulièrement mobilisés lors de l'épidémie de Covid-19 pour les malades les plus critiques. Cet article propose d'analyser l'évolution de la géographie de cet équipement en France à deux échelles d'espace et pour deux types de situations : aux échelles régionale et interurbaine et en périodes de crise sanitaire (année passée) et de réforme hospitalière (10 dernières années). Sur les 10 années passées de réforme sanitaire (2008-2018), des indicateurs courants de stock et densités de lits sont utilisés pour mettre en valeur la dynamique d'équipement en lits de réanimation. Les résultats montrent une stabilité du nombre de lits, mais une baisse des densités, en raison notamment de la croissance de la population. Par ailleurs, une redistribution spatiale des lits de réanimation s'est effectuée tant au niveau des aires urbaines que des régions. Elle a abouti à une concentration des lits dans un plus petit nombre d'aires urbaines et à une convergence des densités de lits au niveau des régions. Pour la situation de crise sanitaire, une mesure inédite de potentiel théorique de transformation de certains lits hospitaliers en lits de réanimation est proposée à partir de la dernière date de recensement des lits hospitaliers (2018). Cette mesure permet d'observer que les aires urbaines et les régions disposent de potentiels distincts. Les régions les moins équipées en lits de réanimation par habitant possèdent les potentiels de transformation les plus élevés.

\section{INDEX}

Mots-clés : Covid-19, lits de réanimation, hôpitaux, régions, aires urbaines 


\section{AUTEURS}

\section{BENOIT CONTI}

LVMT, Université Gustave Eiffel, Ecole des Ponts, Champs-sur-Marne, France,

SOPHIE BAUDET-MICHEL

UMR Géographie-cités, Université de Paris

CHARLÈNE LE NEINDRE

IRDES 